\title{
viewpoint
}

\section{The future of neo-eugenics}

\author{
Now that many people approve theelimination of certain genetically defectivefetuses, is society closer to \\ screening all fetusesfor all known mutations?
}

\section{Armand Marie Leroi}

E ery year, 4.1 million babies are born in the USA. On the basis of the wellknown risk of Down syndrome, about 6,150 of these babies would be expected to suffer from this genetic condition, which is caused by an extra copy of chromosome 21 . In reality, only about 4,370 babies are born with Down syndrome; the others have been aborted during pregnancy. These estimates are based on a prevalence rate of $0.15 \%$ and an abortion rate of about $29 \%$ of fetuses diagnosed with Down syndrome in Atlanta, GA (Siffel et al, 2004), and Hawaii (Forrester $\&$ M erz, 2002)-the only two US locations for which reliable data are available. Data from other regions are similar or even higher: $32 \%$ of D own syndrome fetuses were aborted in Western Australia (Bourke et al, 2005); $75 \%$ in South Australia (Cheffins et al, 2000); 80\% in Taiwan (Jou et al, 2005); and $85 \%$ in Paris, France (Khoshnood et al, 2004). Despite this trend, the total number of babies born with Down syndrome is not declining in most industrialized nations because both the number of older mothers and the conception rate is increasing.

These abortions are eugenic in both intention and effect- that is, their purpose is to eliminate a genetically defective fetus and thus allow for a genetically superior child in a subsequent pregnancy. This is a harsh way of phrasing it; another way is to say that parents just want to have healthy children. Nevertheless, however it is phrased, the conclusion is starkly unavoidable: terminating the pregnancy of a genetically defective fetus is widespread. M oreover, because none of the countries mentioned above coerce parents into aborting deformed fetuses, these abortions-which number many thousands each year-are carried out at the request of the parents, or at least the mothers. This high number of so-called medical abortions shows that many people, in many parts of the world, consider the elimination of a genetically defective fetus to be morally acceptable.

This form of eugenic selection is not confined to Down syndrome, which is characterized by mental retardation, a higher risk of various diseases, and a range of major and minor abnormalities in body structure and function. Fetuses with many disorders detectable by ultrasound in utero are also aborted. Data from the European Surveillance of Congenital Abnormalities shows that between 1995 and 1999 about $40 \%$ of infants with any one of 11 main congenital disorders were aborted in Europe (Garne et al, 2005). Similarly, the International Clearinghouse for Birth Defects Monitoring System (ICBDMS; Rome, Italy) provides data for the eight main industrialized (G8) countries. From this data, I calculate that in $2002,20 \%$ of fetuses with apparent birth defects were aborted in G 8 countries-that is, between 30,000 and 40,000 fetuses. As a result, many congenital disorders are becoming rare (ICBDMS, 2004) and, as they do, infant mortality rates are also declining. In Western Australia, neonatal mortality rates due to congenital deformities declined from 4.36 to 2.75 per 1,000 births in the period from 1980 to

\section{Thishigh number of so-called medical abortions shows that many people... consider the elimination of a genetically defectivefetus to be morally acceptable}

1998. Half of that decline is thought to be due to the increase in abortions of abnormal fetuses (Bourke et al, 2005).

T he widespread acceptance of abortion as a eugenic practice suggests that there might be little resistance to more sophisticated methods of eugenic selection and, in general, this has been the case. Increasingly, prenatal diagnosis of genetic conditions is carried out on the basis of molecular tests for Mendelian disorders. There are few published data on the frequency and consequences of such tests, but a recent survey of genetic testing in Italy showed that about 20,000 fetuses were tested in 2004, mostly for mutations causing cystic fibrosis, Duchenne's muscular dystrophy and Fragile $X$ mental retardation (Dallapiccola et al, 2006). In Taiwan, screens for thalassaemia mutations have caused the live-birth prevalence of this disease to drop from 5.6 to 1.21 per 100,000 births over eight years (Chern et al, 2006).

However, such tests probably do not markedly decrease the mutational burden of a nation's newborns. U sually, a fetus is only tested for a specific mutation when its family medical history indicates that there is a clear risk. If, as must often be the case, parents are oblivious to the fact that they are carriers of a genetic disorder, they will have no reason to undergo a prenatal diagnosis, which is both expensive and invasive. Fetuses are also not tested for de novo mutations. However, given that manyperhaps most-parents want healthy children, should all fetuses be screened for many disease-causing mutations?

It is a question that some geneticists are now asking (Van den Veyver \& Beaudet, 
2006). They point out that comparative genomic hybridization (CGH) microarrays could be used to screen a single embryo or fetus for thousands of mutations. O ne type of CGH microarray that is close to clinical application is designed to detect changes in gene copy number across the whole genome (Vissers et al, 2005). These arrays, which are based on bacterial artificial chromosome (BAC) clones, can detect aneusomies-deletions and duplications- of about 100 kilobases in size. Such aneusomies are found in almost all individuals with no negative consequences, but a minority, which affect dosage-sensitive genes, cause disease. A recent study in which 100 patients with unexplained mental retardation were screened for aneusomies gives some indication of the importance of aneusomies in genetic disorders (de Vries et al, 2005). Most of the copy number changes found in these patients were also found in healthy parents or controls and thus were probably not responsible for the disease; however, ten patients had unique de novo mutations. Therefore, this study identified a likelyalbeit unproven-genetic cause of mental retardation in $10 \%$ of patients; a remarkable result for a single screen.

The virtue of a BAC-based microarray is that it can detect novel, as well as known, deletions and duplications; its limitation is that it misses the point mutations that are the cause of many, perhaps most, genetic diseases. Such mutations presumably account for at least some of the retardation in the 90 patients in whom no aneusomies were detected. At present there is no feasible method of screening the genome of a patient for all possible mutations-at least not without sequencing it. H owever, there is no technical obstacle to constructing an oligo-based micoarray able to detect all known disease-causing mutations.

ow useful would such a microarray be? More precisely, if a geneticist were able to screen a randomly chosen embryo for all known disease genes, what is the probability that he or she would be able to predict a genetic disease should the embryo come to term and live to adulthood? At the time of writing, the Human Gene Mutation Database (HGMD: www.hgmd.cf.ac.uk) identifies 64,251 mutations in 2,362 human genes that impair health. Most of these mutations are individually rare, but collectively they are very common. Indeed, given that there are so many mutations, the probability that an embryo is at risk of a genetic disease caused by at least one of them must be quite high.

An individual's risk of suffering from a genetic disease depends on the mode of inheritance of the disease-autosomal dominant (AD), X-linked recessive (XLR) or autosomal recessive (AR)- and the global frequency of the causal mutation. A survey of 567 disease-causing loci from the $\mathrm{O}$ nline $\mathrm{M}$ endelian Inheritance in $\mathrm{Man}$ database showed that about $59 \%$ are $A D$, $32 \%$ are $A R$, and $9 \%$ are XLR (JimenezSanchez et al, 2001). U sing these percentages with the 64,251 known diseasecausing mutations in HGMD, we can estimate that 37,908 are $A D, 20,560$ are $A R$ and 5,783 are XLR.

\section{... thereis no technical obstacle to constructing an oligo-based micoarray ableto detect all known disease-causing mutations}

To complete our calculation, we need to know the typical global frequencies of each of these three types of mutation. It is surprisingly difficult to obtain global frequency data for disease alleles; however, Reich \& Lander (2001) give the total frequencies of all known disease mutations for 14 monogenic diseases: $4 A D, 3 X L R$, and 7 AR. The HGMD then provides us with the total number of disease-causing mutations known for each of these 14 genes, which ranges from 31 for haemochromatosis to 1,262 for cystic fibrosis.

Using these figures, I have calculated average allelic frequencies (Table 1). The fact that AR mutations are more common than $A D$ or XLR mutations makes sense, as selection acts less intensively on them. Multiplying these numbers by the number of mutations in each inheritance class calculated above, while taking into account the mode of inheritance and assuming global $\mathrm{H}$ ardy-Weinberg equilibrium, I calculate that the probability of predicting an inherited disease in a randomly chosen human embryo is almost $0.4 \%$ (Table 1 ). Therefore, it should be possible to predict a disease in 1 in 252 embryos.

The prediction of a genetic disease in a fetus does not necessarily indicate that it should be aborted. This decision ultimately depends on the strength of the prediction and the nature of the disease, both of
Table1|The probability of predicting a genetic diseasein a random embryo if it were screened for all currently known mutations.

\begin{tabular}{llll}
\hline Inheritance & $\mathbf{n}$ & $\mathbf{p}$ & $\mathbf{F}$ \\
\hline AD & 37,908 & $4.9 \times 10^{-8}$ & $1.9 \times 10^{-3}$ \\
\hline XLR & 5,629 & $2.4 \times 10^{-7}$ & $6.7 \times 10^{-4}$ \\
\hline AR & 20,560 & $2.6 \times 10^{-4}$ & $1.4 \times 10^{-3}$ \\
\hline Sum & 64,097 & & $3.97 \times 10^{-3}$
\end{tabular}

nisthenumber of mutations; pistheaveragefrequency; Fisthefrequency of humans in which a disease can be predicted. To calculateF, I assumeglobal Hardy-Weinberg equilibrium (violation of this owing to population structure tends to inflateF). $F(A D)=n p ; F(A R)=n p^{2}$;

$F(X L R)=0.5 n p(1+p)$. I excluded G6PD from the XLR genes listed by Reich \& Lander (2001), and from the mutation total in theH uman GenomeM utation Database, becausemutant alleles in this geneareso common that its inclusion vastly inflates thenumber of diseased fetuses, even though homozygotes haveonly mild health effects. Mode of inheritance: $A D$, autosomal dominant; $X L R, X$-linked recessive; $A R$, autosomal recessive.

which vary greatly among mutations. A female embryo with a single BRCA1 mutation, which is dominant, has a $68 \%$ probability of developing breast cancer by the age of 80 (Risch et al, 2001). Conversely, an embryo with two copies of the HFE C282Y mutation, which is recessive, has less than a $1 \%$ probability of developing haemochromatosis, a relatively mild blood disease (Beutler et al, 2002). Whether such risks warrant aborting either fetus is a decision to be made by its parents and their clinical advisors, but it should be noted that most of the mutations in the HGMD cause classical Mendelian disorders detected by family linkage studies and so have fairly high penetrance.

\section{... the number of peopleacross the world who have survived a PGD screen must now number tens of thousands}

The estimate of the rate of disease prediction that I have given here is crude, but it is probably conservative. For convenience, I assumed a $\mathrm{H}$ ardy-Weinberg equilibrium, but in isolated populations or populations with a high degree of consanguinity-for instance, much of the Middle East through to Pakistan-the number of disease-causing homozygotes will be higher than my calculations. In addition, the rate of disease prediction will continue to rise as more and more disease-causing mutations are found. In 2005, 7,017 mutations were added to the HGM D-26\% more than in 2004. 


\section{saience\& society}

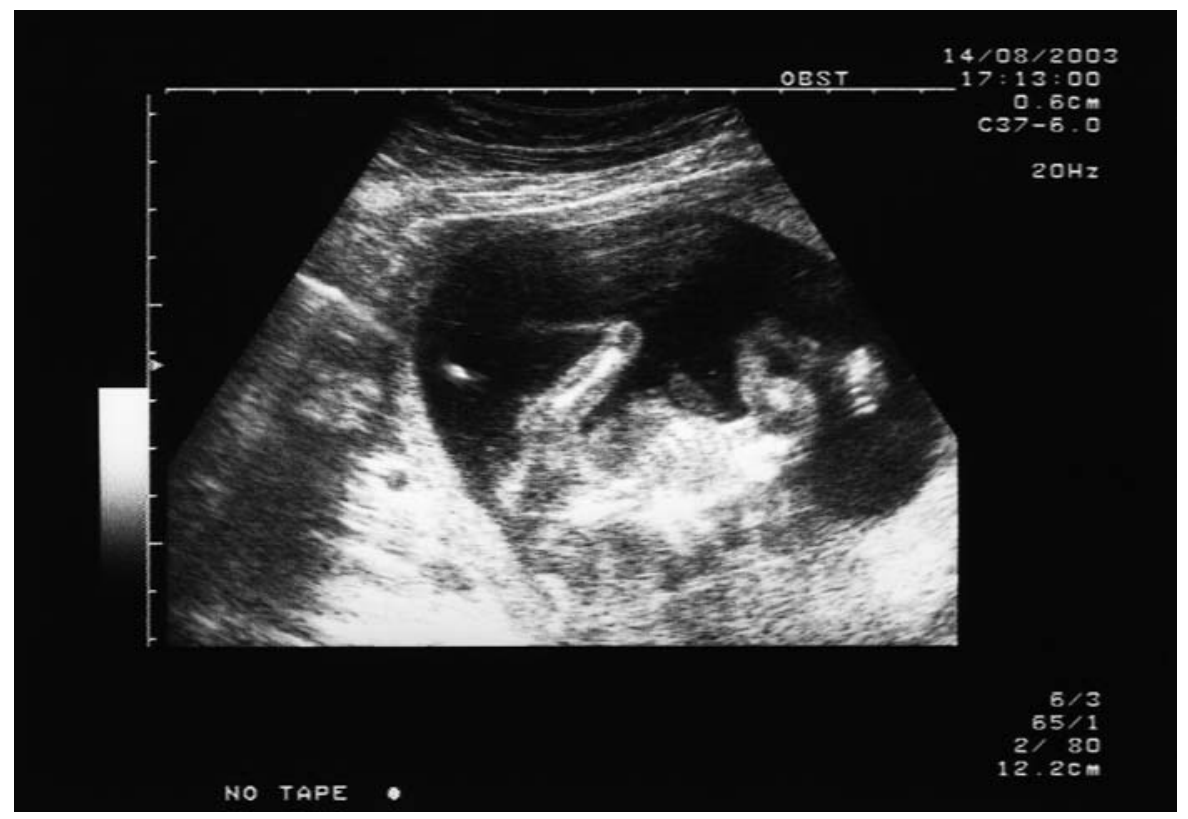

Fig1|Ultrasound scan to amniocentesis test. Amniocentesis is a diagnostic procedureperformed by inserting a needle (seen on the left) through theabdominal wall into theuterus and withdrawing a small amount of fluid from the sac surrounding thefetus. Thetest can detect chromosomal disorders, such as D own syndrome, structural defects, such as spina bifida (open spine, wherethevertebraefail to close), anencephaly (a condition in which thebrain isincompleteor missing), and many rare, inherited metabolic disorders.

o M ediscan/Corbis

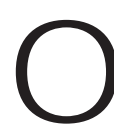
ne impediment to a universal, total prenatal screen for all known mutations is the invasive nature of the procedure-it requires amniocentesis (Fig 1) or chorionic sampling to retrieve cells from the amniotic sac- and the traumatic nature of the treatment, which is therapeutic abortion. Perhaps, then, a total mutation screen will not be used in prenatal diagnosis, but rather in preimplantation genetic diagnosis (PGD). This procedure tests embryos produced by in vitro fertilization (IVF) for chromosomal abnormalities and specific mutations before implantation, by removing a single cell from the embryo at the eight-cell stage. Healthy embryos are then implanted; poor embryos-showing one or several abnormalities-are frozen or discarded. As in prenatal diagnosis, PGD is generally carried out only when a family medical history suggests that the embryo is at risk of a specific disease (Braude et al, 2002). Since its introduction in the mid-1980s, the procedure has spread quickly, although it remains illegal in some countries, such as Germany, which does, however, allow prenatal screens for a range of severe inheritable diseases. Data collected by the European IVF-monitoring Programme for the European Society of
Human Reproduction and Embryology (ESHRE; Grimbergen, Belgium) showed that European nations in 2002, compared with 882 in 2001 (Andersen et al, 2006). There do not seem to be any comparable data for the USA, but given the large number of US IVF clinics offering PGD- and the lack of regulation-the number of people across the world who have survived a PGD screen must now number tens of thousands.

How common will PGD become? Is it possible that one day every citizen of an industrialized nation will have survived, as an embryo, a PGD screen? Most commentators who have considered such a scenario-which was portrayed in the movie GATTACA- do not think so (Silver, 2000). Their main argument is that PGD - and the need to use IVF- is too expensive, inconvenient and limited in application to ever

\section{It seems possible that an increasing number of parents will choose not to subject their children to the vicissitudes of natural conception and the risk of severe genetic disease} 1,563 PGD screens were recorded in 25 become widespread. They have a point: nature has contrived a cheap, easy and enjoyable way to conceive a child; IVF is none of these things.

However, the difficulties might be exaggerated. A course of IVF in the UK costs between $£ 7,000$ and $£ 10,000$ - expensive, but cheaper than a mid-range car, and trivial compared with the costs of raising a child. Conception rates using IVF are generally lower compared with the oldfashioned method, but that is because many of the women who undergo IVF are relatively old (CDC, 2003). For women under 35 who have no fertility problems, the success rate per cycle is greater than $50 \%$, which is comparable to natural monthly conception rates. However, perhaps the most important evidence against the idea that IVF-and PGD - will not catch on is the observation that it al ready has. At present, about $1 \%$ of Americans are conceived using IVF, and each year $4 \%$ of $D$ anes start their life in a petri dish (N yboe Andersen \& Erb, 2006). It seems possible that if the cost of IVF decreases further and the number of PGD screens expands, an increasing number of parents will choose not to subject their children to the vicissitudes of natural conception and the risk of severe genetic disease.

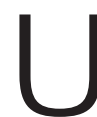
Itimately, the argument for a universal, total mutation screen will be based on its economic costs and benefits. It is too soon to draw up a detailed balance sheet, but we can suggest some numbers. Congenital mental retardation afflicts about 51,000 children annually in the USA; the Centers for Disease Control and Prevention estimate that each afflicted child will cost the US economy \$1 million over the course of his or her life-that is, a collective cost of $\$ 51$ billion (CDC, 2004). This does not include the social and emotional cost that parents assume in raising a mentally disabled child, which all but defy quantification.

Will neo-eugenics spread? Probably. At least it is hard to see what will stop it if, as I claim, it becomes possible to detect all known disease-causing mutations before birth or implantation, if the cost of IVF and PGD declines, and if eugenic screens have clear economic benefits. Some readers might find it peculiar that in this discussion of neoeugenics, I have not considered the ethical or legal implications with which this subject is generally considered to be fraught. Although I do not doubt their importance, I simply have no particular knowledge of them. Peter 
Medawar put it best 40 years ago: "If the termination of a pregnancy is now in question, scientific evidence might tell us that the chances of a defective birth are 100 percent, 50 percent, 25 percent, or perhaps unascertainable. The evidence is highly relevant to the decision, but the decision itself is not a scientific one, and I see no reason why scientists as such should be specially well-qualified to make it" (Medawar, 1966).

\section{REFEREN CES}

Andersen AN, Gianaroli L, Felberbaum R, de Mouzon J, Nygren KG (2006) Assisted reproductive technology in Europe, 2002. Results generated from European registers by ESH RE. Hum Reprod 21: 1680-1697

Beutler E, Felitti VJ, Koziol JA, Ho NJ, GelbartT (2002) Penetrance of 845G -->A (C282Y) HFE hereditary haemochromatosis mutation in the USA. Lancet 359:211-218

Bourke J, Bower C, Blair E, Charles A, Knuiman M (2005) The effect of terminations of pregnancy for fetal abnormalities on trends in mortality to one year of age in Western Australia. Paediatr Perinat Epidemiol 19: 284-293

Braude P, Pickering S, Flinter F, O gilvie CM (2002) Preimplantation genetic diagnosis. $\mathrm{N}$ at Rev Genet 3: 941-953

CDC (2003) Assisted Reproductive Technology Success Rates. $N$ ational Summary and Fertility Clinic Reports 2003. Atlanta, GA, U SA: Centers for Disease Control and Prevention

CDC (2004) Economic costs associated with mental retardation, cerebral palsy, hearing loss, and vision impairment-U nited States, 2003. M M WR M orb M ortal W kly Rep 53: 57-59

Cheffins T et al (2000) The impact of maternal serum screening on the birth prevalence of Down's syndrome and the use of amniocentesis and chorionic villus sampling in South Australia. BJO G 107: 1453-1459

Chern JP, Lin KH, SuYN, Lu MY, Jou ST, Lin DT, Wang SC, Lin KS (2006) Impact of a national $\beta$-thalassemia carrier screening program on the birth rate of thalassemia major. Pediatr Blood Cancer 46: 72-76

Dallapiccola B, Torrente I, M orena A, Dagna-Bricarelli F, M ingarelli R (2006) Genetic testing in Italy, year 2004. Eur J H um Genet 14: 911-916

de Vries BB et al (2005) Diagnostic genome profiling in mental retardation. Am J H um Genet 77: 606-616

Forrester M B, M erz RD (2002) Epidemiology of D own syndrome (Trisomy 21), H awaii, 1986-97. Teratol 2002 65:207-112

Garne E et al (2005) Prenatal diagnosis of severe structural congenital malformations in Europe. U Itrasound O bstet Gynecol 25: 6-11

ICBD M S (2004) Annual Report 2004 with Data for 2002. Rome, Italy: International Centre on Birth Defects

Jimenez-Sanchez G, Childs B, Valle D (2001) H uman disease genes. $N$ ature 409: 853-855

Jou HJ, Kuo YS, Hsu JJ, Shyu MK, Hsieh TT, H sieh FJ (2005) The evolving national birth prevalence of D own syndrome in Taiwan. A study on the impact of second-trimester maternal serum screening. Prenat Diagn 25: 665-670

Khoshnood B, De Vigan C, VodovarV, G oujard J, $G$ offinet $F$ (2004) A population-based evaluation of the impact of antenatal screening for D own's syndrome in France, 1981-2000. BJO G 111: 485-490

M edawar PB (1966) Science and the sanctity of life. In (1982) Pluto's Republic, pp 311-323. O xford, UK: O xford U niversity Press

Nyboe Andersen A, Erb K (2006) Register data on Assisted Reproductive Technology (ART) in Europe including a detailed description of ART in Denmark. Int J Androl 29: 12-16
Reich DE, Lander ES (2001) O n the allelic spectrum of human disease. Trends G enet 17: 501-510

Risch HA et al (2001) Prevalence and penetrance of germline BRCA 1 and BRCA 2 mutations in a population series of 649 women with ovarian cancer. Am J H um G enet 68: 700-710

Siffel C, Correa A, Cragan J, Alverson CJ (2004) Prenatal diagnosis, pregnancy terminations and prevalence of D own syndrome in Atlanta. Birth D efects Res A Clin M ol Teratol 70: 565-571

Silver LM (2000) Reprogenetics: third millennium speculation. EMBO Rep 1: 375-378

Van den Veyver IB, BeaudetAL (2006) Comparative genomic hybridization and prenatal diagnosis. Curr O pin O bstet Gynecol 18: 185-191

Vissers LE, Veltman JA, van Kessel AG, Brunner HG (2005) Identification of human disease genes by whole genome CGH arrays. Hum M ol Genet 14 (RI2): R215-R213

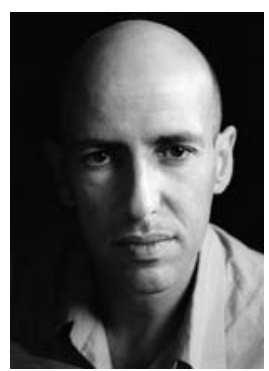

Armand Marie Leroi is a researcher at Imperial College, London, UK, and theauthor of Mutants: On theForm, Varietiesand Errors of the Human Body.

E-mail:a.leroi@imperial.ac.uk

doi:10.1038/sj.embor.7400860 